Casos Clínicos

Arch. Esp. Urol., 59, 6 (640-644), 2006

\section{A PROPÓSITO DE UN CASO: PRIAPISMO DE ALTO FLUJO.}

\author{
Anna Bujons, X. Pascual, J. Martí, P. De la Torre, H. \\ Sarquella y Humberto Villavicencio.
}

Servicio de Urología Fundación Puigvert. Barcelona. España.

Resumen.- OBJETIVO: Se presenta el caso clínico de un paciente con priapismo de 4 años de evolución, su diagnóstico y posterior manejo terapéutico.

MÉTODOS: Se revisa en la literatura, etiología, formas de presentación y discusión de los hallazgos clínicos, radiológicos y diagnóstico diferencial.

RESULTADOS:El paciente es sometido a embolización de la arteria pudenda interna con excelentes resultados.

CONCLUSIONES: La embolización de la arteria pudenda interna es un tratamiento eficaz para la resolución de un priaprismo de alto flujo por una fístula arterio-venosa.

Palabras clave: Priapismo. Alto flujo.
Summary.- OBJECTIVE: We report the case of a patient consulting with a four-year history of priapism, its diagnosis and subsequent therapeutic management.

METHODS: We reviewed the literature for etiology, types of presentation, discussion of the clinical and radiological findings, and differential diagnosis.

RESULTS: The patient underwent the embolization of the internal pudendal artery with excellent results.

CONCLUSIONS: The embolization of the internal pudendal artery is an effective treatment for the resolution of high flow priapism secondary to arterial venous fistula.

Keywords: Priapism. High flow.

\section{INTRODUCCIÓN}

Se define priapismo como la erección peneana mantenida y patológica, sin relación con el deseo sexual, habitualmente provocada por un fallo hemodinámico en el seno de los cuerpos cavernosos, que altera el equilibrio entre el aporte arterial y su drenaje venoso; puede afectar a todas las edades.

Existen dos tipos de priapismo: el venoso o de bajo flujo, que es característicamente doloroso debido a su componente isquémico y por lo tanto, puede conllevar peor pronóstico, debido al riesgo de fibrosis peneana e impotencia, y el arterial o alto flujo, no isquémico y por tanto indoloro y menos frecuente. Los dos tipos difieren tanto en la etiología, fisiopatología, clínica y tratamiento.

Se presenta el caso de un paciente en estudio de priapismo de 4 años de evolución, su diagnóstico y posterior manejo terapéutico.

\section{CASO CLÍNICO}

Paciente varón de 47 años de edad, sin hábitos tóxicos ni antecedentes patológicos de interés, acude a la consulta de andrología por presentar erecciones prolongadas no dolorosas de aproximadamente 4 años de evolución tras traumatismo perineal cerrado con el manillar de una bicicleta.

En la exploración física se observan cuerpos cavernosos aumentados de consistencia, no dolorosos a la palpación, sin palpar pulsos anómalos. Sensibilidad peneana conservada. Testes móviles en ambas bolsas escrotales $y \sin$ alteraciones.

Como exploraciones complementarias se le realiza ecodoppler penenano: vascularización cavernosa derecha 


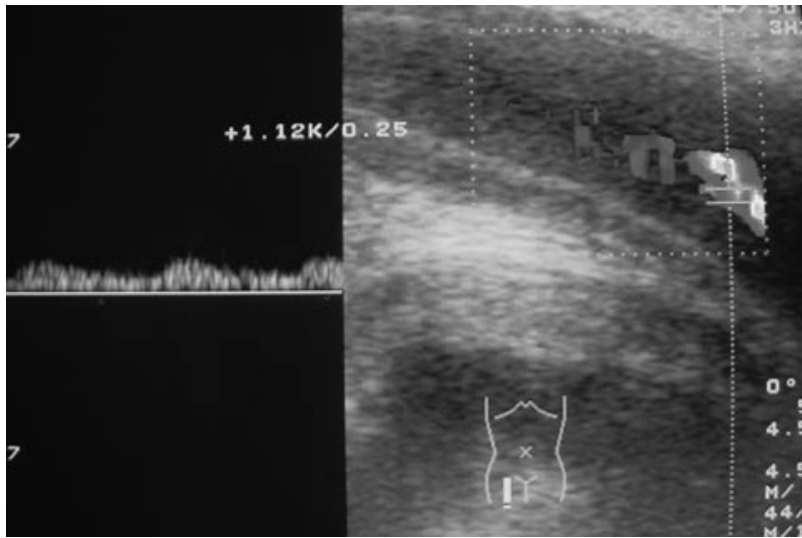

FIGURA 1. Flujo sinusoidal anterógrado y turbulento en cuerpo cavernoso lesionado.

aparentemente conservada; en la porción más proximal del cuerpo cavernoso izquierdo se observa formación anecoica $(2 \times 1.8 \times 1.5 \mathrm{~cm})$ con flujo turbulento en su interior compatible con fístula arteriovenosa (FAV) de larga duración (Figura 1).

Con la orientación diagnóstica de Priapismo de alto flujo se decide realización de Arteriografía pudenda con anestesia local confirmándose FAV y posterior embolización de la misma mediante 2 coil $3 \times 5$. (Figuras 2 y 3 ).

Tras la embolización el paciente evoluciona favorablemente con detumecencia peneana completa y erecciones normales. Actualmente está asintomático.

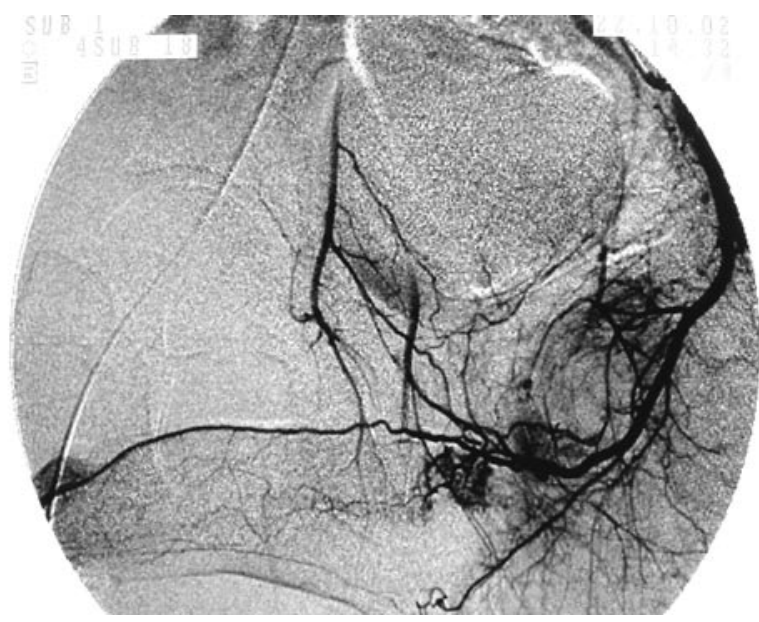

FIGURA 2. FAV arteria pudenda izquierda cuerpo cavernoso.

\section{DISCUSIÓN}

Los mecanismos reguladores de la detumecencia y mantenimiento de la flacidez peneana, a nivel local son las terminaciones nerviosas adrenérgicas (neurotransmisor noradrenalina), que provocan vasoconstricción peneana y contracción del músculo trabecular, con posterior reducción del flujo arterial y colapso de los espacios lacunares permitiendo su drenaje venoso. El péptido endotelina y las prostaglandinas $\left(\mathrm{PGF}_{2}\right.$, tromboxano) también están implicados, mientras que los factores favorecedores de la erección son la prostaglandina $\mathrm{PGE}_{2}$ y NO, que causan relajación de la musculatura trabecular y vasodilatación peneana.

\section{Clasificación}

El priapismo se clasifica en veno-oclusivo o de bajo flujo, caracterizado por un estado de isquemia, y arterial o de alto flujo, no isquémico.

El priapismo de origen veno-oclusivo es más frecuente. Es el tipo de priapismo que entraña mayor potencial de causar alteración permanente de la disfunción eréctil. Se produce una alteración en el retorno venoso por obstrucción parcial o completa del drenaje de los cuerpos cavernosos impidiendo flujo de entrada de sangre arterial, estableciéndose una estado isquémico mantenido de los cuerpos cavernosos $(1,2)$.

La dificultad del drenaje venoso puede ser debida a una compresión extravascular aunque puede acabar teniendo un efecto intravascular por formación de trombos sanguíneos en las vénulas de drenaje como consecuencia de la estasis sanguínea, o de causa intravascular por trombosis venosa, añadiendo un efecto extravascular por edema del tejido trabecular.

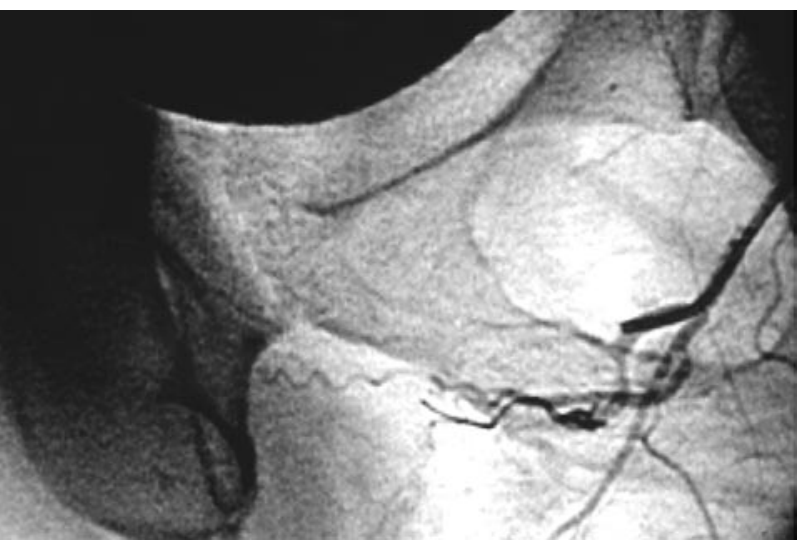

FIGURA 3. Comprobación de la oclusión tras embolización FAV. 


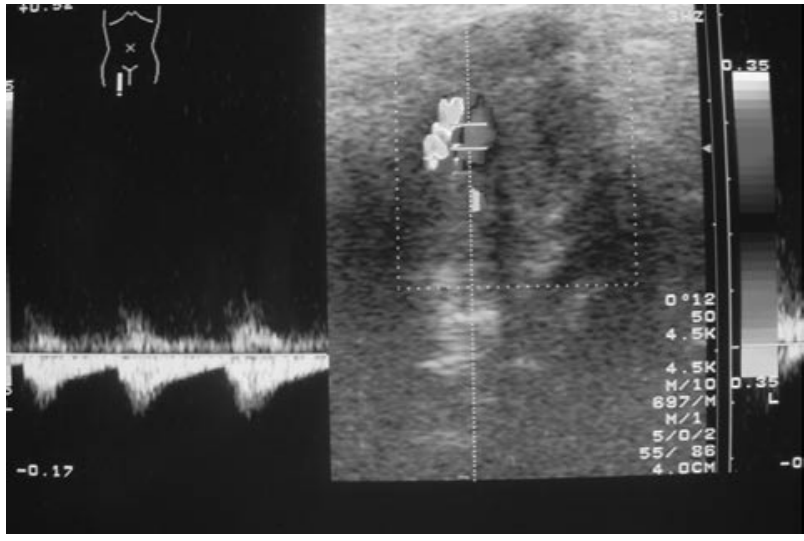

FIGURA 4. Flujo turbulento arterial en área de lesión de cuerpo cavernoso.

Tras la isquemia, se produce en los cuerpos cavernosos hipoxia severa y acidosis, causando depresión en la contractilidad del músculo trabecular y en consecuencia imposibilidad de la desactivación del mecanismo venooclusivo, impidiendo el retorno venoso.

El Óxido Nítrico (NO) y las prostaciclinas $\left(\mathrm{PGI}_{2}\right)$ están implicados en el mecanismo de la erección y detumecencia peneana. Durante la erección, el flujo arterial de los cuerpos cavernosos facilita la síntesis de $\mathrm{NO}$ y $\mathrm{PGI}_{2}$, mecanismos reguladores de la interacción entre la sangre acumulada y la pared trabecular. Durante el priapismo veno-oclusivo, la hipoxia y en consecuencia la isquemia mantenida altera la síntesis de $\mathrm{NO}$ y $\mathrm{PGI}_{2}$ alterándose el equilibrio homeostático y facilitándose la agregación plaquetaria y la adhesión y activación de células inflamatorias, provocando trombosis de las vénulas con bloqueo vascular persistente, pudiendo llevar a la muerte celular y posterior fibrosis en el proceso de reparación de tejido, en casos de evolución de priapismo de más de 12 horas de evolución. (2)

Hay varias causas de origen veno-oclusivo. La causa más frecuente es de origen extravascular y de causa farmacológica, por la inyección intracavernosa de fármacos vasoactivos en el tratamiento de la disfunción eréctil. Otros fármacos implicados pueden ser psicofármacos (antipsicóticos y antidepresivos), antihipertensivos, o por compresión de tumor sólido. Las de origen intravascular pueden ser provocados por hemoglobinopatías como la Anemia de las células falciformes, nutrición parenteral, procesos malignos con hipercelularidad y tumores sólidos.

El paciente presenta una erección dolorosa, agitado y con antecedentes de erecciones prolongadas después de mantener relaciones sexuales o nocturnas. En la exploración física el pene se encuentra en un estado de erección completa.

El priapismo de origen arterial es menos frecuente, no isquémico, y por lo tanto, indoloro, suele ser secundario en

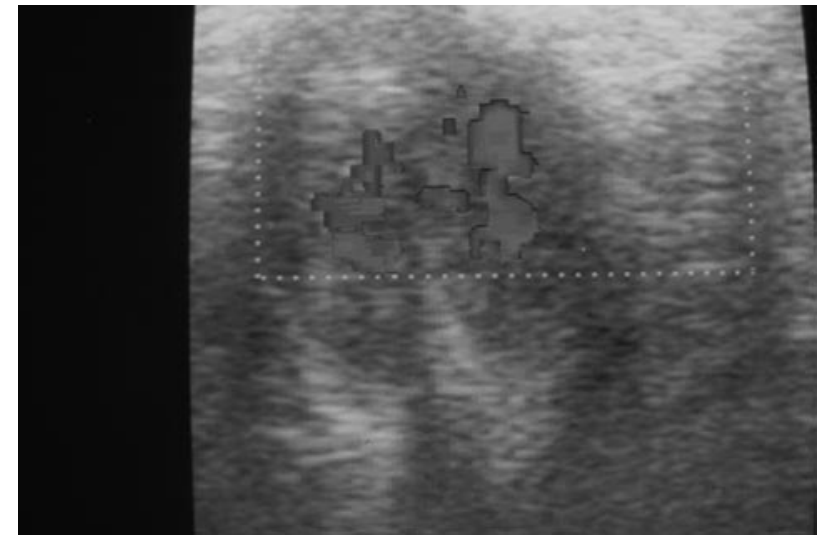

FIGURA 5. Inversión de flujo en cuerpo cavernoso lesionado.

la mayoría de los casos, a un traumatismo penenano o perineal y conlleva mejor pronóstico.

El flujo arterial en los espacios lacunares está regulado por las arterias helicinas, que se hallan en estado de constricción en el pene flácido creándose un gradiente de presión entre la arteria cavernosa y el estado lacunar, produciéndose en el priapismo arterial una alteración en este mecanismo de regulación, ya que la arteria cavernosa $o$ una de sus ramas, lacerada por un traumatismo, forma una fístula arterio-lacunar, estableciéndose una ingurgitación anterógrada por hiperaflujo arterial, manteniéndose normal el drenaje de los cuerpos cavernosos. Al no existir déficit de flujo arterial no hay isquemia ni necrosis de los tejidos. Se produce un flujo turbulento de alta velocidad y junto a la hiperoxigenación favorece la síntesis de NO por el endotelio causando vasodilatación de todo el cuerpo cavernoso.

Casi siempre está causado por un traumatismo en zona perineal o penenana, aunque tambíen puede ser provocado por inyecciones intracavernosas de prostaglandinas, en casos en que la aguja insertada en el cuerpo cavernoso lacere la pared arterial, o en casos de cirugía de revascularización peneana.

Clínicamente, el priapismo arterial se manifiesta como una erección peneana indolora, continua que aparece de forma inmediata o tardía tras el traumatismo. En la Exploración física se observa una erección generalmente incompleta $(60-70 \%)$, a veces puede acompañarse de hematoma o tumefacción peneana, escrotal o perineal asociada por el traumatismo.

\section{Diagnóstico}

El priaprismo se considera una urgencia médica.

El objetivo ante un priapismo es determinar el origen venoso o arterial ya que el manejo terapéutico es distinto y retornar la flacidez peneana con el manteniemiento de la capacidad eréctil del pene. 

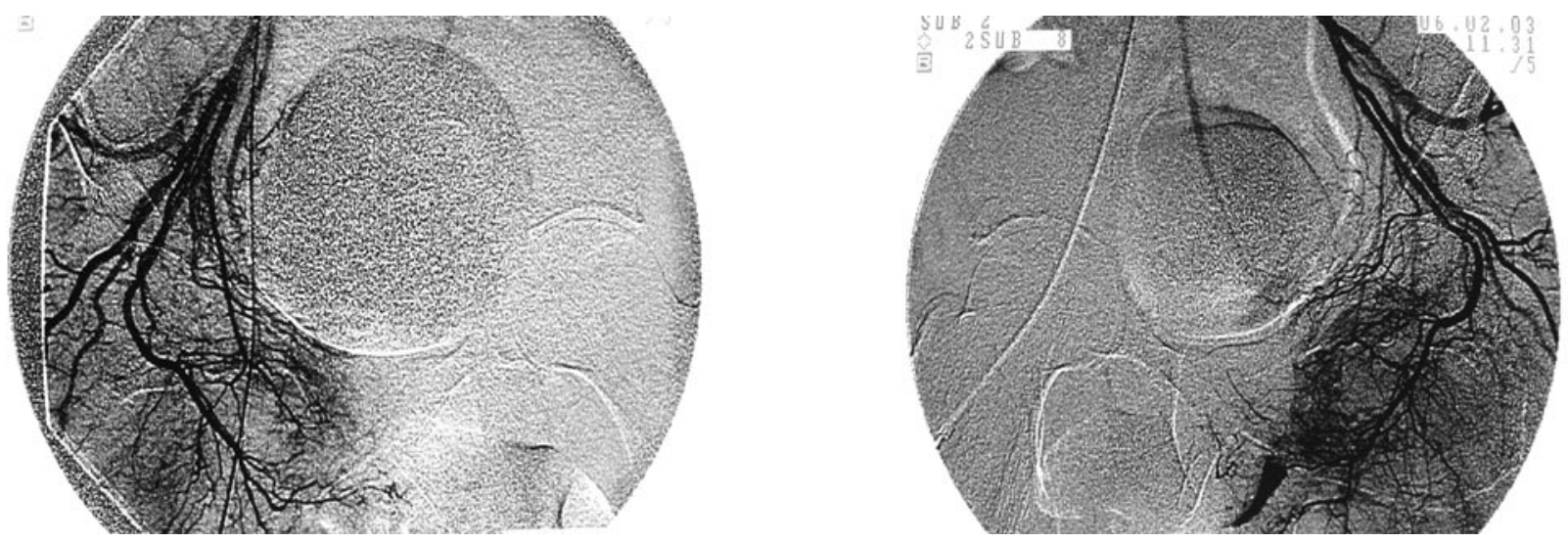

FIGURA 6. Asimetría comparativa arterias pudendas.

En primer lugar, la Historia clínica y los síntomas nos orientan a una $u$ otra forma de priapismo. Si el paciente está en tratamiento farmacológico con antihipertensivos, antipsicóticos u otros que pueden estar asociados a priapismo se debe interrumpir.

A continuación, es determinar el flujo arterial de los cuerpos cavernosos mediante una gasometría de los cuerpos cavernosos y el eco-doppler.

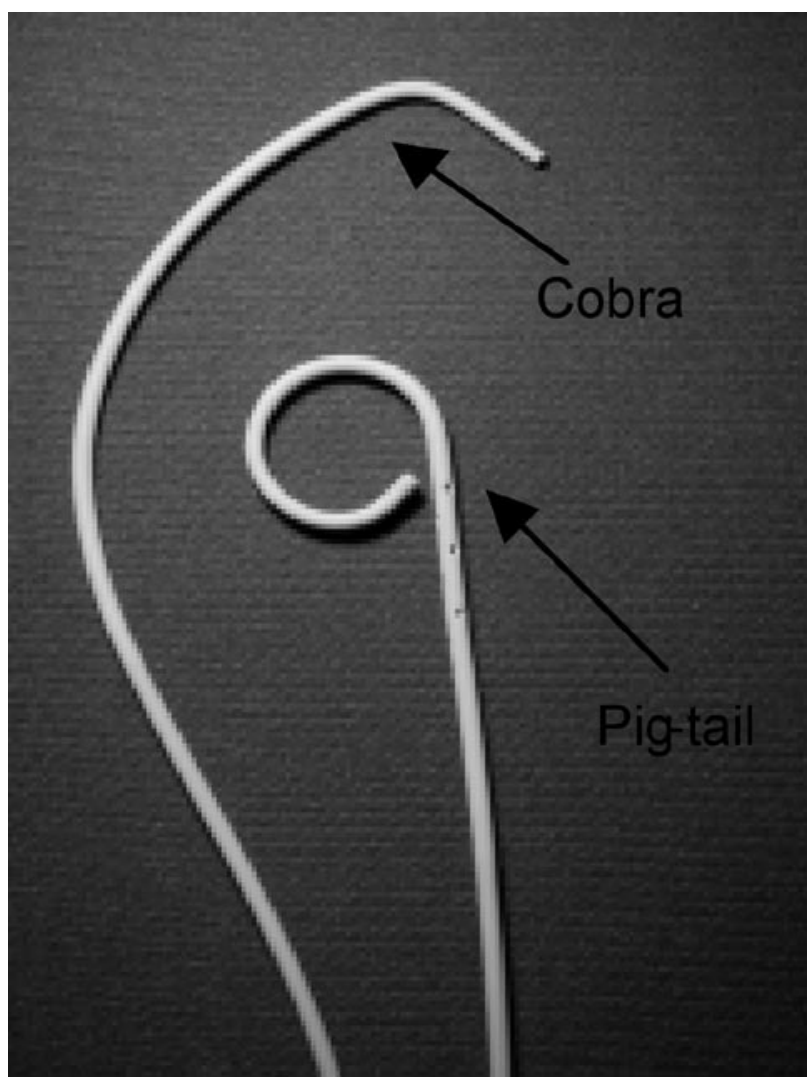

FIGURA 7. El catéter cobra permite alcanzar los tramos más distales de la arteria pudenda. El catéter pig-tail se utiliza para el estudio segmentario arterial pelviano.
El eco-doppler es la técnica de elección en el diagnóstico diferencial de un priapismo indoloro por ser rápida, indolora, inocua y no invasiva, pudiendo detectar, identificar y localizar la lesión causal del priapismo, que puede corresponder a una fístula arteriovenosa, un pseudoaneurisma o a una asimetría del flujo sistodiastólico entre el lado lesionado y el indemne. Se podrá observar si existe circulación cavernosa, presencia de área de flujo turbulento (Figura 4) e inversión de flujo (Figura 5) y presencia de registros arteriales $\operatorname{Res}(4,5)$.

En la gasometría arterial, si la $\mathrm{PO}_{2}$ es de $100 \mathrm{mmHg}$ y la $\mathrm{PO}_{2}$ del cuerpo cavernoso es de $60 \mathrm{mmHg}$ se trata de un priaprismo de origen veno-oclusivo, mientras que si $\mathrm{PO}_{2}$ del cuerpo cavernoso es la misma que la gasometría arterial el origen del priapismo es de alto flujo.

En algunos casos se realiza Pudendografía, que tiene dos fases: Se realiza primero una fase segmentaria y una selectiva. El objetivo es observar una posible asimetría de ambas arterias pudendas (Figura 6) y la existencia de una posible fístula con posterior embolización de la misma.

Para realización de la pudendografía segmentaria se utiliza catéter pig-tail $6 \mathrm{Fr}$ y para la selectiva se utiliza catetér cobra (Figura 7).

\section{Tratamiento del priapismo}

Ante un paciente con priapismo de origen veno-oclusivo de pocas horas de evolución, el drenaje de la sangre de los cuerpos cavernosos se realiza con agujas de calibre grueso (19G a 14G) en surco balanoprepucial con o sin anestesia local o espinal. El drenaje de la sangre se realiza lentamente a la vez que se ejerce una compresión o masaje del pene. Si el pene queda flácido, se cierra la aguja de drenaje y se espera 15-20 minutos para ver que retorne el priapismo.

Si la erección reaparece, se procede a la inyección intracavernosa de agonistas alfa-adrenérgicos (fenilefrina, etilefrina) monitorizando la presión arterial, por lo que pueden producirse crisis hipertensivas. 
Si el priapismo tiene una duración prolongada, el drenaje de los cuerpos cavernosos puede ser difícil por el aumento de la viscosidad sanguínea debido a un periodo prolongado de isquemia, pudiéndose irrigar primero el cuerpo cavernoso con suero salino para facilitar el drenaje. En caso de fracaso de los alfa-adrenérgicos, se deben realizar fístulas de conexión entre los cuerpos cavernosos con sistemas de baja resistencia como glande, cuerpo esponjoso, vena safena, con el objetivo de aumentar el flujo de drenaje de los espacios lacunares y reducir la presión intracavernosa para que pueda aumentar el flujo de sangre oxigenada. No obstante, la reoxigenación de los cuerpos cavernosos isquémicos causa la liberación de metabolitos reactivos de oxígeno con potencial de dañar los cuerpos cavernosos; para evitarlo, antes de la realización del drenaje de los cuerpos cavernosos se pueden administrar fármacos antioxidantes como la vitamina $E$ intramuscular y vitamina $C$ intravenosa. Después de la realización de las fístulas no se deben aplicar vendajes compresivos en el pene porque pueden reducir el flujo arterial y aumentar la isquemia, provocando necrosis peneana.

Ante un priapismo de origen arterial el paciente refiere un antecedente traumático, presenta una erección no dolorosa, hay buen flujo de sangre en el doppler y la gasometría intracavernosa demuestra valores de sangre arterial. El tratamiento no tiene porque ser inmediato, ya que no existe isquemia, pudiéndose diferir.

Hay casos en que la erección disminuye de forma progresiva hasta la remisión completa al cabo de días o semanas, aunque lo habitual es que se mantenga durante meses.

El tratamiento de elección de un priapismo arterial es la embolización arterial selectiva, que se realiza simultáneamente a la pudendografía.. Se puede utilizar material rebsorvible con trombos autólogos, esponjas de gelatina o espongostán, con posterior recanalización de la arteria tras reabsorción. Las ventajas de esta técnica son el escasa trauma peneano y la posibilidad de evaluación inmediata de los resultados por vía angiográfica. También puede utilizarse material irreabsorvible como el bucrylato o el coil de platino. Las complicaciones son escasas, como la infección del coágulo autólogo, y se consigue una detumecencia rápida.
En casos de laceración bilateral el tratamiento se debe hacer con dos tiempos.

Cuando la embolización no es posible, se realiza ligadura de la arteria lacerada, aunque puede provocar daño del tejido cavernoso durante la exploración y que la arteria no se recanalice.

La principal complicación del priapismo es la impotencia secundaria, siendo el pronóstico peor en el priapismo venoso.

\section{CONCLUSIONES}

Ante un paciente con priapismo el objetivo principal es determinar su origen venoso o arterial por su distinto manejo terapéutico, ya que el de causa veno-oclusivo es isquémico, su tratamiento son los alfa-adrenérgicos y es una urgencia médica; su demora podría inducir a una disfunción eréctil permanente, mientras que el priapismo arterial no es isquémico, su tratamiento puede diferirse y se trata con la embolización.

El eco-doppler es la técnica de elección para el diagnóstico diferencial debido a su inocuidad y rapidez.

\section{BIBLIOGRAFÍA y LECTURAS RECOMENDADAS (*lectura de interés $y^{* *}$ lectura fundamental)}

**1. MARTÍNEZ DEL CASTILLO, M.L.; MIGUÉLEZ LAGO C.; GARCÍA MÉRIDA, M. y GALIANO DURO, E. y cols.: "Autoembolización espontánea en un niño con priapismo postraumático de alto flujo. Actas Urol. Esp. 24:820, 2000.

*2. SÁENZ DE TEJADA, I. y GORMAN: Priapismo: Fisiopatología y tratamiento médico.

3. GUDINCHET.; FOURNIER, D. y JICHSLINSKI, P.: "Traumatic priapism in a child: valuation with color flow doppler sonography. The J. Urol. 148:380, 1992.

4. FELDSTEIN, V.A.: "Posttraumatic "high flow" priapism evaluation with color flow doppler sonography". J. Ultrasound Med. 12:589, 1993. 